\title{
Endovascular Treatment of Distal Posterior Cerebral Artery Infectious Aneurysm
}

\author{
Manoj Bohara $P h D^{1}$, Prakash Bista $M C^{2} h^{2}$ \\ ${ }^{1,2}$ Department of Neurosurgery, Hospital for Advanced Medicine and Surgery (HAMS), Kathmandu, Nepal
}

\section{Abstract}

Intracranial infectious aneurysms are rare entities accounting for approximately 1- $6 \%$ of all cerebral aneurysms and have high propensity of rupture associated with mortality. The principal risk factor is infective endocarditis and the management includes antimicrobial treatment with or without obliteration of the aneurysm by microsurgical or endovascular means.

We present a young patient with intracranial infectious aneurysm who was successfully treated with endovascular coil embolization. A 20-year-old female with a history of rheumatic heart disease presented with subarachnoid hemorrhage due to rupture of IIA associated with infective endocarditis. Cerebral angiogram revealed right distal posterior cerebral artery aneurysm. Echocardiography showed vegetation in the mitral valve and blood culture was positive for Enterococcus faecalis. Antibiotic treatment was administered for 6 weeks. The follow-up angiogram showed an enlarging aneurysm. So, the patient underwent endovascular coil embolization of the aneurysm preserving the parent artery. There were no post-procedure deficits.

Intracranial infectious aneurysm should be considered as a differential diagnosis in a patient with infective endocarditis presenting with focal neurological deficits or altered consciousness. Early diagnosis and individualized approach are the key to successful treatment and endovascular treatment is an effective modality for such lesions.

Key words: Coil embolization, Infective endocarditis, Intracranial infectious aneurysm, Mycotic aneurysm

\section{Introduction}

I ntracranial infectious aneurysms (IIAs), also referred to as mycotic aneurysms, are rare entities accounting for approximately 1-6\% of all cerebral aneurysms and have

Access this article online
Website: https://www.nepjol.info/index.php/NJN
DOI: https://doi.org/10.3126/njn.v17i3.33127
HOW TO CITE

Bohara M, Bista P. Endovascular Treatment of Distal Posterior Cerebral Artery Infectious Aneurysm. NJNS. 2020;17(3):50-54

${ }^{1}$ ORCID id: 0000-0002-3157-7334

${ }^{2}$ ORCID id: 0000-0002-4811-6964

\section{Address for correspondence:}

Dr. Manoj Bohara

Hospital for Advanced Medicine and Surgery (HAMS),

Mandikhatar Road, Kathmandu, Nepal

E-mail: boharamanoj111@gmail.com

Phone: +977-9851164662

Copyright $(92020$ Nepalese Society of Neurosurgeons (NESON)

ISSN: 1813-1948 (Print), 1813-1956 (Online)

(i) (8) This work is licensed under a Creative Commons Attribution-Non Commercial 4.0 International License. high propensity of rupture associated with mortality risk of up to $80 \%$. $^{1,2}$ The major risk factor for development of IIAs is infective endocarditis (IE). The management of IIAs includes proper antibiotic treatment with or without the obliteration of the aneurysm by microsurgical or endovascular approaches. Owing to the fragile nature of the affected parent artery and the aneurysm wall as well as the common locations being distal, the treatment of these aneurysms pose a challenge compared to the classical saccular aneurysms. Parent artery occlusion, by microsurgical or endovascular techniques, is usually performed as a radical solution but may lead to significant neurological deficits. ${ }^{3}$

We here report a 20 -year-old female with rheumatic heart disease and IE who developed subarachnoid hemorrhage due to rupture of a distal posterior cerebral artery (PCA) aneurysm, which was successfully treated with endovascular coil embolization preserving the parent artery. To our knowledge, this is the first case report of IIA treated by endovascular technique in Nepal where neurointerventional treatment has recently been available.

\section{Case Report}

A 20-year-old female with history of rheumatic heart disease(RHD)undermedications Phenoxymethylpenicillin 


\section{Coiling of Mycotic Aneurysm}

and Enalapril and also history of renal and splenic infarction presented to our Emergency Department with altered consciousness which was preceded by severe headache and projectile vomiting. On examination, Glasgow Coma Scale was 13/15. Computed Tomography (CT) scan of brain revealed subarachnoid hemorrhage (SAH) in the right posterior temporal region (Figure 1A). CT angiography showed right distal PCA (parieto-occipital branch) aneurysm (Figures 1B and 1C) suggestive of IIA. Echocardiography demonstrated vegetation measuring $1.2 \mathrm{~cm}$ in posterior mitral leaf, with dilated left atrium and severe mitral regurgitation. Thus, a diagnosis of infective endocarditis with ruptured cerebral IIA was made. Blood culture was positive for Ampicillin-resistant Enterococcus Faecalis and intravenous Teicoplanin and Gentamicin were administered for 6 weeks with serial CT angiogram monitoring. The patient recovered clinically with resolution of SAH; however, the right distal PCA aneurysm increased in size (from $3.4 \mathrm{~mm} \times 4.2 \mathrm{~mm}$ to $5.1 \mathrm{~mm} \times 4.2 \mathrm{~mm}$ ) on follow up angiography. To avoid the risk of re-rupture, we planned for endovascular coil embolization of the aneurysm.

Owing to the saccular aneurysm with a good neck, we planned for embolization of the aneurysm alone, preserving the parent artery if possible. Under general anesthesia and systemic heparinization, a 6F right femoral sheath was inserted. Injection through a 6F Envoy guiding catheter (Cordis, Miami Lakes, FL, USA) placed in the left vertebral artery revealed the aneurysm on right distal PCA (Figures 2A and 2B). Selective microcatheter injection was done via Excelsior SL-10 microcatheter (Stryker, Fremont, CA, USA) (Figures 2C and 2D), which was advanced over a 0.014-inch Transend micro-guidewire (Stryker, Fremont, CA, USA) into the right distal PCA aneurysm. Embolization was performed through the microcatheter using three Axium Prime Extra Soft detachable coils sized $3 \mathrm{~mm} \times 8 \mathrm{~cm}$ helix, $2.5 \mathrm{~mm}$ x $8 \mathrm{~cm}$ helix and $1 \mathrm{~mm} \times 4 \mathrm{~cm}$ 3D (ev3/Covidien, Irvine, CA, USA). Post-embolization angiography revealed obliteration of the aneurysm with parent artery preservation (Figures $3 \mathrm{~A}$ and $3 \mathrm{~B}$ ). The patient had no peri-procedural complications. Magnetic resonance imaging (MRI) and MR angiography (MRA) performed a week after embolization showed obliteration of the aneurysm with coils and no infarction. She was discharged on Phenoxymethylpenicillin and Enalapril as prescribed by cardiologists. No antiplatelet was used in this case because the parent artery was preserved during coiling. On the latest follow-up after 6 months of the procedure, she had no deficits and was independently carrying out her daily activities. MRA at 6-month followup, showed good coil packing with no recurrence of the aneurysm (Figure 3C).

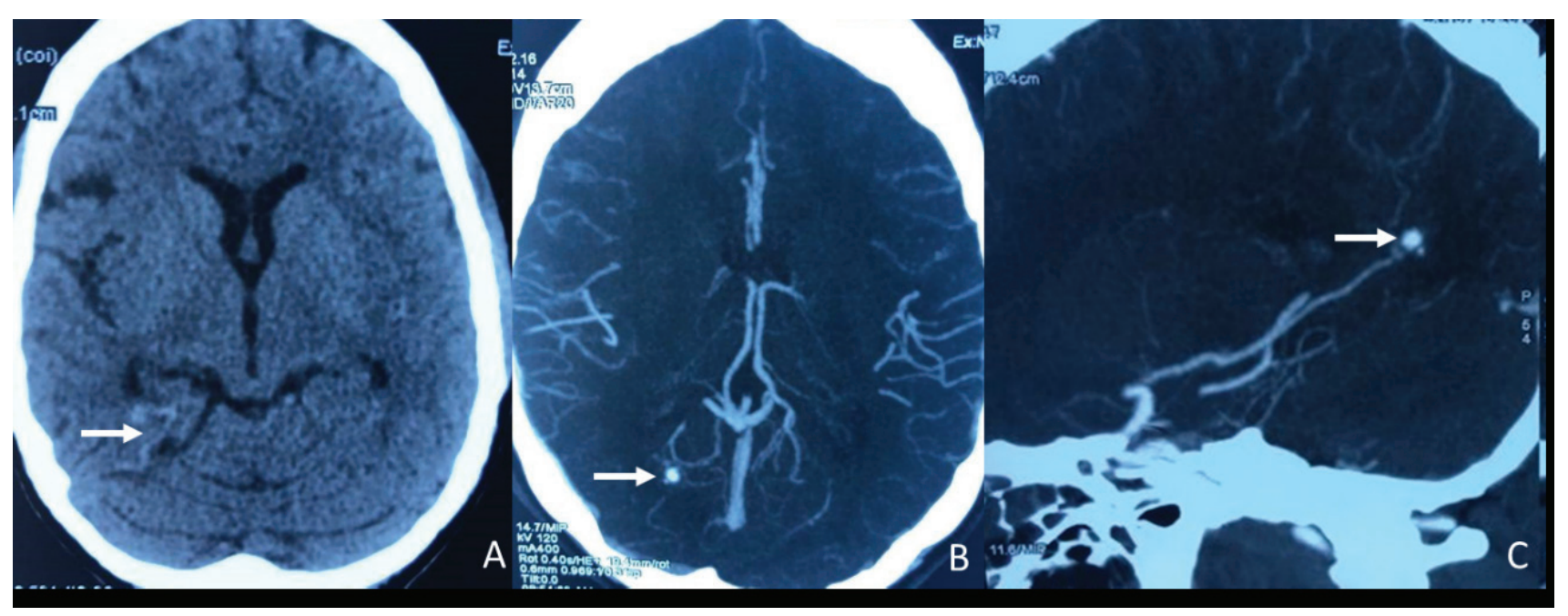

Figure 1: Computed tomography scans showing subarachnoid hemorrhage (A; white arrow) due to rupture of the right distal posterior cerebral artery aneurysm (B and $C$; white arrow). 


\section{Bohara et al}

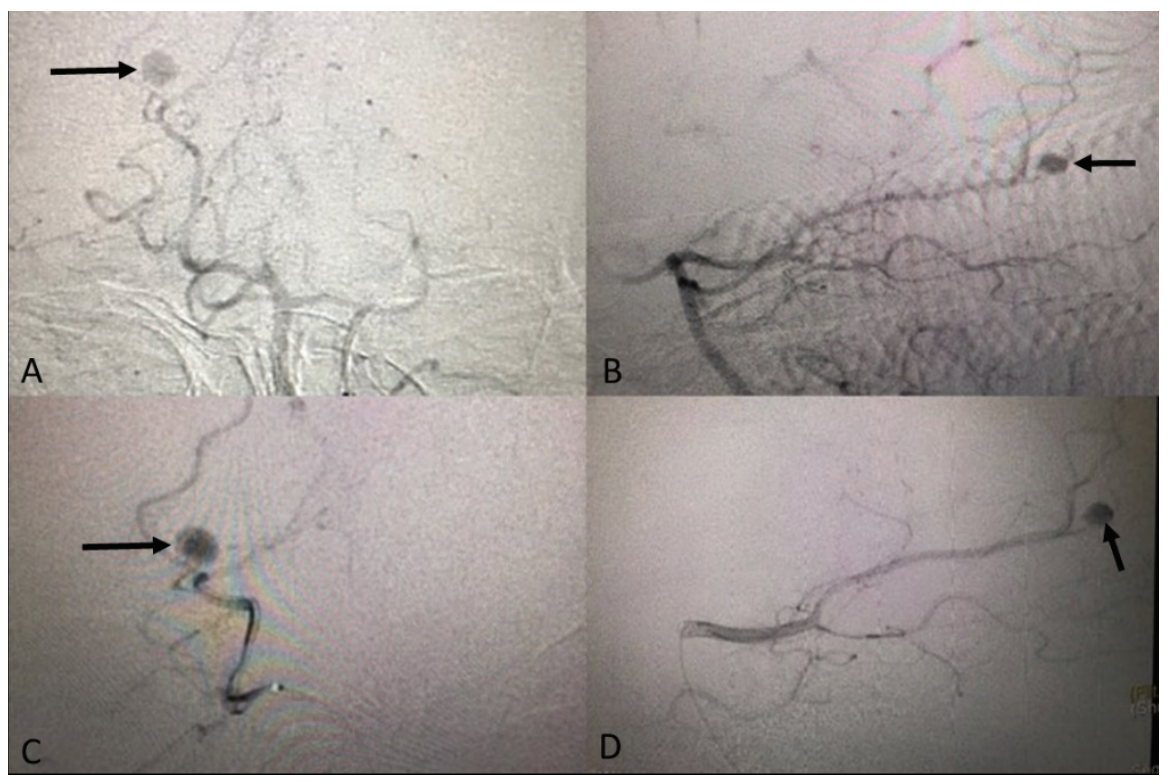

Figure 2: Conventional digital subtraction angiography via catheter placed in left vertebral artery (A and B) and selective angiography via microcatheter placed in right posterior cerebral artery (PCA) (C and D) revealing the aneurysm of distal right PCA (black arrows).

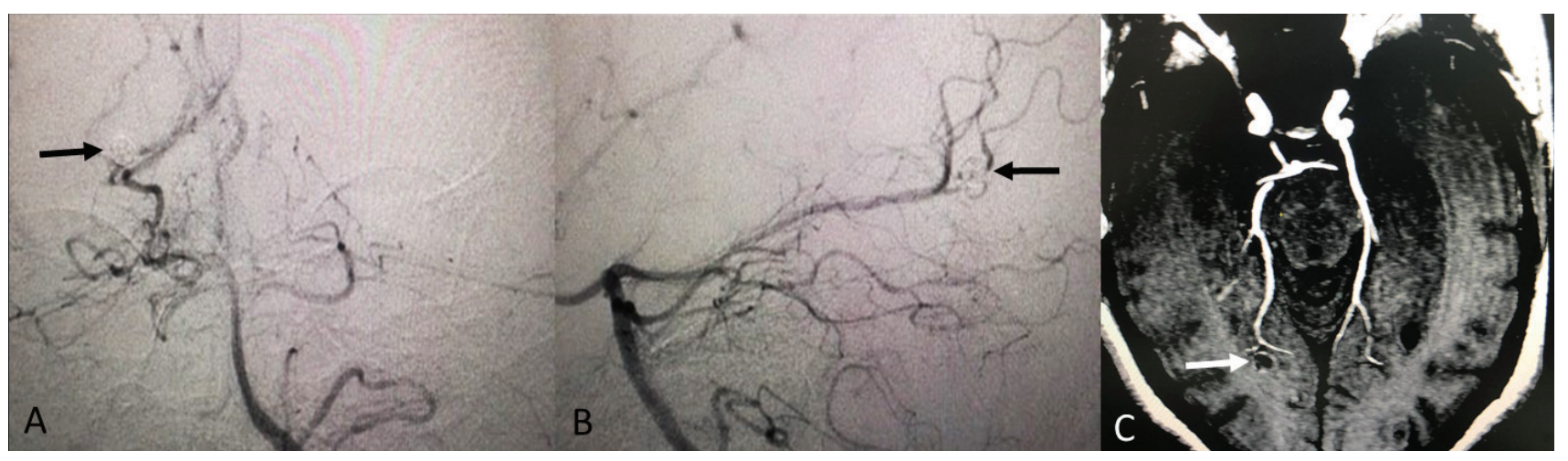

Figure 3: Post-embolization immediate catheter angiography (A and B) revealed obliteration of the aneurysm with coils (black arrows) and parent artery preservation. Magnetic resonance angiography at 6-month follow-up (C) revealed no recurrence of the aneurysm (packed coils denoted by white arrow).

\section{Discussion}

IIAs are pseudoaneurysms of infectious origin, the principal risk factor being infective endocarditis in about $80 \%$ of the patients. Other less common etiologies include meningitis, brain abscess, subdural empyema, cavernous sinus thrombophlebitis, sinus infection and osteomyelitis of skull. ${ }^{4}$

Two mechanisms are widely accepted regarding the development of IIAs: intra-arterial and extra-arterial spread of infection. Intra-arterial spread of septic emboli into distal cerebral vessels, generally arising from an infected valve in IE represents the most common cause of IIAs. Two different patho-mechanisms have been hypothesized concerning the intra-arterial spread; the vasa vasorum hypothesis, which suggests that IIA inflammatory responses originate in the adventitia and spreads inwards to the intima. Hydrostatic pulsation and the pressure against the weakened wall of the vessel leads to aneurysm development. ${ }^{5}$ The other mechanism postulated is embolic hypothesis, where infection spreads centrifugally from intima to adventitia. Septic emboli occludes the arterial lumen and causes inflammation of the wall making it fragile and leads to aneurysm development. Initially such patients may present with infarction from the septic emboli. ${ }^{6,7}$ Extra-arterial spread from surrounding infections as in meningitis, cavernous sinus thrombophlebitis and sinus infections has also been reported in the formation of IIAs. ${ }^{8,9}$

Early diagnosis of IIAs is important to prevent significant morbidity and mortality. The presence of predisposing infection, ischemic or hemorrhagic stroke 


\section{Coiling of Mycotic Aneurysm}

in a young patient, history of fever, headache or focal neurological deficits in a patient with IE, presence of multiple aneurysms are some of the features which can arise high suspicion of IIAs. ${ }^{8}$ In our case, a young patient aged 20 years with rheumatic heart disease and history of renal and splenic infarction presented with decreased consciousness, which immediately alerted us to the cerebrovascular involvement. Typically, angiography shows distal location, fusiform, irregular outline, multiplicity and poorly defined neck. Sequential imaging usually shows a change in size and morphology of the aneurysm. Digital subtraction angiography (DSA) is considered the gold standard to diagnose IIAs as they are smaller and more distally located, so may not be revealed by computed tomography angiography (CTA) or magnetic resonance angiography (MRA). ${ }^{10}$ A comprehensive review by Ducruet et al showed that middle cerebral artery (MCA) was the most common location $(57.4 \%$ ) whereas PCA accounted for only $17.6 \%$ of IIAs. The morphology was fusiform in $52.5 \%$ whereas saccular in $41 \%{ }^{1}$ Thus, a saccular IIA of distal PCA as in our case is rare.

The treatment approach for IIAs is variable among clinicians and is dependent on the patient's hemodynamic and rupture status. No randomized controlled trials or prospective cohort studies have evaluated the various treatment approaches to IIAs in infective endocarditis. Usually a combination of antimicrobial, surgical and/or endovascular approaches is used. A 6-week antimicrobial therapy tailored to the culture results is considered the standard medical treatment for IIAs. Serial angiographic studies are recommended during this period for unruptured cases as the IIAs may regress, disappear, persist, enlarge or rupture. If the aneurysm resolves or regresses, surgical or endovascular intervention is not necessary and medical treatment can be continued. However, if the size is unchanged or increased, more aggressive treatment approach should be followed, considering the high morbidity and mortality rate in case of rupture. ${ }^{11,12}$

Surgical or endovascular intervention may be considered for ruptured cases, although some still manage such cases conservatively and the timing of intervention is also a matter of debate. Some clinicians adopt the antibiotic therapy with "wait and watch" policy even with ruptured aneurysms, as there have been reports of resolution of ruptured aneurysms with conservative management alone. ${ }^{8,13}$ While, others have suggested that ruptured IIAs should be immediately secured by microsurgical or endovascular techniques in order to prevent significant morbidity and mortality. ${ }^{11}$ Singla et al in their study of the nationwide database demonstrated that only $18.6 \%$ underwent invasive treatment (surgery or embolization) out of 393 patients with the primary diagnosis of IIA (92\% with SAH). The overall mortality rate was high (36.7\%) with ruptured IIAs; it was higher in medically treated patients compared with those who underwent invasive treatment $(26.7 \%$ vs $15.1 \%){ }^{12}$ In our case, we kept our patient on antimicrobial regimen along with angiographic follow-up, which showed increase in the size of the distal PCA aneurysm. Thus, we decided to treat her by endovascular technique.

Whether microsurgical or endovascular therapy is appropriate depends on individual cases and various factors like clinical status and aneurysm morphology. ${ }^{13}$ The presence of large hematoma with mass effect and increased intracranial pressure may favor treatment by craniotomy. However, microsurgical treatment, including clipping or trapping with/without bypass, is very challenging for IIAs due to unfavorable morphology and location in most of the cases. Recently, endovascular treatment has been seen as an effective and safer approach for IIAs. As most of the IIAs do not have good neck and the aneurysm wall is very fragile, parent artery occlusion (PAO) may be needed which may result in significant neurological deficits if it supplies eloquent areas. In a review by Gross et al, only $24 \%$ of the IIAs were successfully treated with coil embolization alone and the majority required PAO. ${ }^{14}$ PAO using permanent liquid embolics or detachable balloons have also been described in literature. ${ }^{15,16}$ In our case, owing to the saccular aneurysm with good neck, we successfully performed coil embolization of the aneurysm preserving the parent artery with no post-procedure deficits.

\section{Conclusion}

We herein presented a rare case of ruptured IIA associated with IE in a young female, treated with endovascular coil embolization preserving the parent artery. This paper further emphasizes that IIA should be considered as a differential diagnosis in a patient with IE presenting with focal neurological deficits or altered consciousness. Early diagnosis and individualized approach are the key to successful treatment. With the advent of advanced neuro-endovascular techniques, these lesions have been successfully treated with minimal complications in recent times.

Conflict of Interest: None

Source(s) of support: None 


\section{References}

1. Ducruet AF, Hickman ZL, Zacharia BE, Narula R, Grobelny BT, Gorski J, et al. Intracranial infectious aneurysms: a comprehensive review. Neurosurg Rev. 2010;33(1):37-46. https://doi.org/10.1007/s10143009-0233-1

2. Alawieh A, Chaudry MI, Turner RD, Turk AS, Spiotta AM. Infectious intracranial aneurysms: a systematic review of epidemiology, management, and outcomes. J Neurointerv Surg. 2018;10(7):70816. https://doi.org/10.1136/neurintsurg-2017-013603

3. Champeaux C, Walker N, Derwin J, Grivas A. Successful delayed coiling of a ruptured growing distal posterior cerebral artery mycotic aneurysm. Neurochirurgie. 2017;63(1):17-20. https://doi. org/10.1016/j.neuchi.2016.10.005

4. Mayer SA, Bederson JB. Infectious intracranial aneurysms. In: Winn HR, ed. Youmans Neurological Surgery. 6th ed. Saunders: Philadelphia; 2011. p. 3972-7. https://doi.org/10.1016/B978-1-4160-53163.00382-8

5. Molinari GF, Smith L, Goldstein MN, Satran R. Pathogenesis of cerebral mycotic aneuryms. Neurology 1973;23:325-32. https://doi.org/10.1212/ wnl.23.4.325

6. Krapf H, Skalej M, Voigt K. Subarachnoid hemorrhage due to septic embolic infarction in infective endocarditis. Cerebrovasc Dis. 1999;9:1824. https://doi.org/10.1159/000015952

7. Kanai R, Shinoda J, Irie S, Inoue K, Sato T, Tsutsumi Y.A case of embolic stroke imitating atherothrombotic brain infarction before massive hemorrhage from an infectious aneurysm caused by Streptococci. J Stroke Cerebrovasc Dis. 2012;21:910.e13-16. https:/doi. org/10.1016/j.jstrokecerebrovasdis.2011.11.001

8. Esenkaya A, Duzgun F, Cinar C, Bozkaya H, Eraslan C, Ozgiray E, et al. Endovascular treatment of intracranial infectious aneurysms. Neuroradiology.
2016;58(3):277-84. https://doi.org/10.1007/s00234015-1633-2

9. Hurst RW, Judkins A, Bolger W, Chu A, Loevner LA. Mycotic aneurysm and cerebral infarction resulting from fungal sinusitis: imaging and pathologic correlation. AJNR Am J Neuroradiol. 2011;22:85863. PMID: 11337328.

10. Hui FK, Bain M, Obuchowski NA, Gordon S, Spiotta AM, Moskowitz S, et al. Mycotic aneurysm detection rates with cerebral angiography in patients with infective endocarditis. J Neurointerv Surg. 2015;7(6):449-52. https://doi.org/10.1136/ neurintsurg-2014-011124

11. Chapot R, Houdart E, Saint-Maurice JP, Aymard A, Mounayer C, Merland JJ. Endovascular treatment of cerebral mycotic aneurysms. Neuroradiology. 2002; 222:389-96. https://doi.org/10.1148/ radiol.2222010432

12. Singla A, Fargen K, Blackburn S, Neal D, Martin TD, Hess PJ, et al. National treatment practices in the management of infectious intracranial aneurysms and infective endocarditis. J Neurointerv Surg. 2015;0:16. https://doi.org/10.1136/neurintsurg-2015-011834

13. Peters PJ, Harrison T, Lennox JL. A dangerous dilemma: management of infectious intracranial aneurysms complicating endocarditis. Lancet Infect Dis. 2006; 6(11):742-8. https://doi.org/10.1016/ S1473-3099(06)70631-4

14. Gross BA, Puri AS. Endovascular treatment of infectious intracranial aneurysms. Neurosurg Rev. 2013;36:11-19. https://doi.org/10.1007/s10143-0120414-1

15. Grandhi R, Zwagerman NT, Linares G, Monaco EA 3rd, Jovin T, Horowitz M, et al. Onyx embolization of infectious intracranial aneurysms. J Neurointerv surg. 2014;6:353-6. https://doi.org/10.1136/ neurintsurg-2013-010755

16. Yuen T1, Laidlaw JD, Mitchell P. Mycotic intracavernous carotid aneurysm. J Clin Neurosci. 2004;11(7):771-5. jocn.2004.02.004 